Georgia State University

ScholarWorks @ Georgia State University

$11-8-2016$

\title{
Selecting, Adapting, and Implementing Evidence-based Interventions in Rural settings: An Analysis of 70 community examples
}

Tina Anderson Smith

Tanisa Foxworth Adimu

Amanda Phillips Martinez

Karen Minyard

Follow this and additional works at: https://scholarworks.gsu.edu/ghpc_articles

\section{Recommended Citation}

Smith, Tina Anderson; Adimu, Tanisa Foxworth; Martinez, Amanda Phillips; and Minyard, Karen, "Selecting, Adapting, and Implementing Evidence-based Interventions in Rural settings: An Analysis of 70 community examples" (2016). GHPC Articles. 139.

https://scholarworks.gsu.edu/ghpc_articles/139

This Article is brought to you for free and open access by the Georgia Health Policy Center at ScholarWorks @ Georgia State University. It has been accepted for inclusion in GHPC Articles by an authorized administrator of ScholarWorks@Georgia State University. For more information, please contact scholarworks@gsu.edu. 


\section{PROJECT MUSE*}

Selecting, Adapting, and Implementing Evidence-based Interventions in Rural Settings: An Analysis of 70 Community Examples

Tina Anderson Smith, Tanisa Foxworth Adimu, Amanda Phillips Martinez, Karen Minyard

Journal of Health Care for the Poor and Underserved, Volume 27, Number 4, November 2016 Supplement, pp. 181-193 (Article)

Published by Johns Hopkins University Press

DOI: https://doi.org/10.1353/hpu.2016.0179

$\Rightarrow$ For additional information about this article https://muse.jhu.edu/article/634886 


\title{
Selecting, Adapting, and Implementing Evidence- based Interventions in Rural Settings: An Analysis of 70 Community Examples
}

\author{
Tina Anderson Smith, MPH \\ Tanisa Foxworth Adimu, MPH \\ Amanda Phillips Martinez, MPH \\ Karen Minyard, $\mathrm{PhD}$
}

\begin{abstract}
Objective. This paper explores how communities translate evidence-based and promising health practices to rural contexts. Methods. A descriptive, qualitative analysis was conducted using data from 70 grantees funded by the Federal Office of Rural Health Policy to implement evidence-based health practices in rural settings. Findings were organized using The Interactive Systems Framework for Dissemination and Implementation. Results. Grantees broadly interpreted evidence-based and promising practices, resulting in the implementation of a patchwork of health-related interventions that fell along a spectrum of evidentiary rigor. The cohort faced common challenges translating recognized practices into rural community settings and reported making deliberate modifications to original models as a result. Conclusion. Opportunities for building a more robust rural health evidence base include investments to incentivize evidence-based programming in rural settings; rural-specific research and theory-building; translation of existing evidence using a rural lens; technical assistance to support rural innovation; and prioritization of evaluation locally.
\end{abstract}

Key words: Rural health, rural health services, rural population, evidence-based models, evidenced-based model adaptation, program implementation, implementation science.

$\mathrm{T}$ he U.S. Health Resources and Services Administration's Federal Office of Rural Health Policy's (FORHP) Rural Health Care Services Outreach (Outreach) Grant Program supports innovations in health outreach and service delivery in rural and frontier areas nationwide. Funding can be used to meet a broad range of health care needs, including health promotion and disease prevention; expanding oral and mental health services; and case management for patients with chronic illnesses. These three-year projects address the needs of a wide range of population groups including low-income

TINA ANDERSON SMITH is affiliated with Anderson Smith Consulting in Atlanta, GA. TANISA FOXWORTH ADIMU and AMANDA PHILLIPS MARTINEZ are Senior Research Associates at Georgia Health Policy Center in Atlanta, GA. KAREN MINYARD is the Director of the Georgia Health Policy Center in Atlanta, GA. Please send correspondence to Amanda Phillips Martinez, MPH, Senior Research Associate, Georgia Health Policy Center, 55 Park Place, Rm 877, Atlanta, GA 30303-2813; Phone: (404) 413-0293; Email: aphillipsmartinez@gsu.edu. 
populations, the elderly, pregnant women, infants, adolescents, rural minority populations, and rural populations with special health care needs.

For the 2012-2015 Outreach grant cycle, FORHP required applicants to propose projects that were evidence-based and/or reflective of promising practices. This focus on applying research- and experience-based models signaled a strategic shift within the Outreach program from investing largely in demonstration projects to funding the spread of empirically-grounded programming into rural settings. The purpose of this approach was to avoid reinvention, with the ultimate goal of improving efficiency, maximizing outcomes, and positioning grantees for the greatest possible impact.

A theoretical framework for translation. Identifying effective interventions is a first step. Transferring and maintaining such programs in real-world settings is a long and complex journey. To facilitate bridging research and practice, particularly in public health, Wandersman and colleagues describe an interrelated set of processes, activities, and dynamics necessary to move effective programs, practices, or policies more rapidly into community practice. ${ }^{1-3}$ This conceptual model, referred to as the Interactive Systems Framework for Dissemination and Implementation (ISF), takes a broad view of the contextual influences and activities that facilitate the implementation of evidence-based innovations in real-world settings. As depicted in Figure 1, ISF comprises three linked systems: (1) Synthesis and Translation System, which distills scientific information about what works and presents it in a way that has the potential to inform action; (2) Innovation Support System, which mediates the other systems by building technical and adaptive capacity and readiness through training, technical assistance, tools, and quality assurance; and (3) Delivery System, comprising frontline stakeholders and practitioners actually implementing the evidence-based innovations. The ISF positions these three systems within a larger environment-funding, macro-policy, socioeconomic climate and existing research/theory-that affects whether and how effectively evidence-based practice is ultimately introduced into community settings.

The extent to which evidenced-based programs are compatible with community settings - at the macro socio-cultural, political, and economic level as well as at the local delivery system level-is well-documented in the broader literature as having a significant impact on the implementation process as well as program outcomes. ${ }^{4-7}$ However, the distinctive characteristics of rural settings are less often the focus of published studies. This gap in information demonstrates a need for researchers, program practitioners, funders, and supporting organizations to consider the implications of rural settings on evidence-based health practice translation. Exploration of these rural dynamics can inform future rural health program design and delivery.

Given current limitations of the rural implementation science literature, the 20122015 Outreach cohort provided an opportunity to study the translation of evidencebased and promising practices specifically in rural settings. The study focused on four questions:

- What range of proven practices are being implemented in rural settings?

- What dynamics affect translation and implementation?

- How and why are evidence-based and promising practices adapted to rural contexts? 


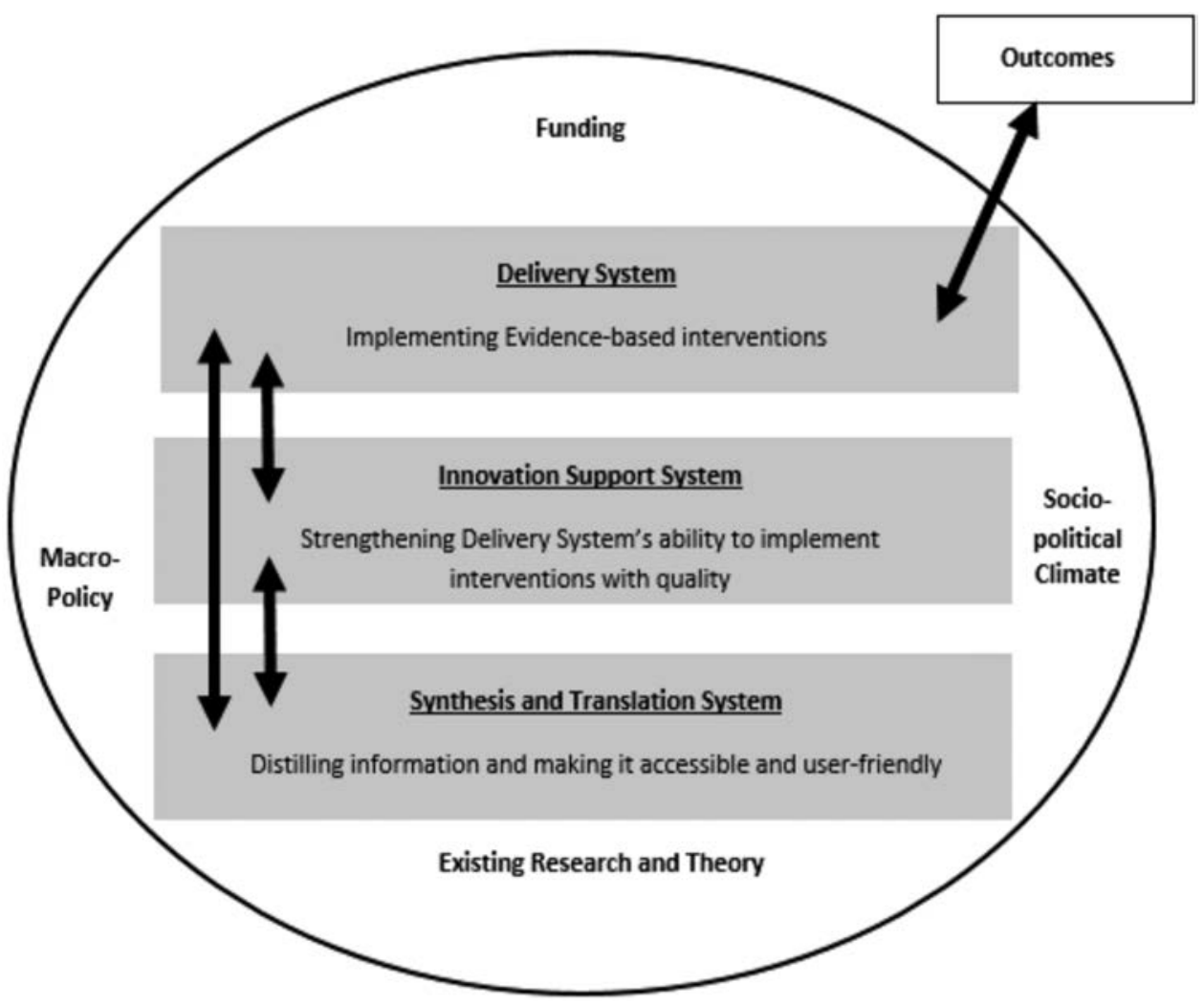

Figure 1. Adapted Interactive Systems Framework for Dissemination and Implementation. Source: Adapted from Wandersman. ${ }^{14}$ The ISF model has been simplified for the purpose of this paper. More comprehensive and more recent versions exist. However, such detail is beyond the scope of this paper.

- What resources support selection, adaptation and implementation in rural environments?

The ISF provides a relevant conceptual lens through which to assess the findings of this study for several reasons: (1) the ISF has been widely cited in the literature and is increasingly applied in practice by the Centers for Disease Control and Prevention, other federal agencies, researchers, and community practitioners; ${ }^{2,3,8-11}$ (2) the model's systems perspective enhances its relevance to a broad range of stakeholders; and (3) the ISF framework blends research-to-practice models with community-centered, practice models, meaning it recognizes the importance of both having research inform practice and practice inform research.

\section{Methods}

Population studied. Seventy delivery systems, representing rural and frontier areas in 36 states, were funded through FORHP's Outreach grant program from 2012 to 2015. Funding was awarded to a lead agency (a public or non-profit private entity) located in 
a non-metropolitan county or in a rural census tract of a metropolitan county. While the majority of agencies were health care organizations (federally qualified health centers, community clinics, and hospitals), FORHP also funded other entities such as churches, school districts, public health departments, and non-profit organizations. The Outreach program requires the participation of a minimum of two additional community partners in grant activities as advisors, service providers, referral sources, and implementing partners. The cohort of grantees implemented a diverse array of program activities intended to improve rural health and health care in a range of clinical focus areas (e.g., chronic disease prevention and management, care coordination, oral health, and maternal child health).

Data collection. Data were collected through extensive document review. Sources included structured final grant reports submitted by all grantees at the close of the grant cycle and summary reports of exit interviews conducted by technical assistance providers with grant program coordinators and other key staff representing each grantee. The final grant report was structured to capture grantees' program implementation experiences as well as any adjustments made to the original program design in response to challenges that arose throughout the three-year grant period.

Technical assistance providers contracted by FORHP completed exit interviews with all 70 grantees. The interviews were designed to complement the submitted final reports. Interviews were transcribed and interview summaries provided additional detail from grantee program staff on the process of identifying, implementing, adapting, and evaluating their evidence-based or promising practice approaches.

Data analysis. Thematic content analysis identified patterns in the data. Two researchers read each report and grant reports and interview summaries were categorized by program focus areas. Researchers identified themes related to the environmental context within which the FORHP-funded programs were implemented; the range of ways that grantees operationalized evidence-based and promising practices when developing their program approach; and the challenges and facilitating factors related to implementation. A second round of data analysis tested themes and further identified patterns within the thematic domains. The resulting patterns were discussed and reconciled during regular data analysis meetings attended by the research team.

\section{Results}

Data analysis revealed the following themes and patterns:

- Grantees broadly interpreted the terms "evidence-based practices" and "promising practices," resulting in the implementation of interventions that fall along a spectrum of evidentiary rigor.

- The majority of grantees applied multiple models and practices within one grant initiative, resulting in hybrid programs or mash-up interventions.

- Grantees faced similar challenges translating evidence-based and promising practices in rural settings.

- Most grantees reported adapting referenced models to overcome challenges and tailoring programs to meet community-specific needs and interests. 
- Grantees received innovation support, including guidance from original evidencebased model sites and technical assistance from an FORHP contractor.

A broad interpretation of evidence-based and promising practices. "Evidencebased programs" and "promising models" are largely terms of art-meaning practitioners interpret them differently across disciplines and fields. In the Funding Opportunity Announcement for the 2012-2015 Outreach grant cycle, FORHP used the following definitions to reinforce the idea that interventions should be grounded in evidence of what works:

Evidence-based programs are those that are developed from scientific evidence and/ or have been found to be effective based on the results of rigorous evaluations. ${ }^{12} \mathrm{~A}$ 'promising model' is defined as one with at least preliminary evidence of effectiveness in small-scale interventions or for which there is potential for generating data that will be useful for making decisions about taking the intervention to scale and generalizing the results to diverse populations and settings. ${ }^{13}$

Nonetheless, grantees construed the terms to include a broad range of concepts and approaches, resulting in the implementation of interventions that fell along a spectrum of evidentiary rigor (Figure 2).

On the left end of the spectrum are indisputable evidence-based practices-timetested interventions built on cumulative, credible evidence of effectiveness in multiple settings. The practices required to achieve the expected outcomes have been carefully articulated by program developers and evaluators and packaged for replication.

The midsection of the spectrum in Figure 2 consists of two distinct types of promising practices operationalized by the grantees. The first subset of promising practices includes well-documented approaches, guidelines, and frameworks that are widely recognized or endorsed by reputable organizations such as the American Heart Association and agencies such as the National Institutes of Health. Such interventions included clinical content and protocols; generally applied concepts and service approaches (e.g., community health workers, medical home models, and telehealth services); and process-oriented frameworks, such as service learning and motivational interviewing. The second type of promising practices involved grantees' replication or more rigorous evaluation of their own interventions, which enjoyed some previous success. In addition to replication and expansion, participants reported using Outreach grant funds to support more rigorous evaluation of the impacts of their programs in new and different settings, ultimately testing the extent to which various approaches might meet the evidence-based standard.

On the far right end of the spectrum in Figure 2 are less well-documented reference programs that grantees said inspired their intervention designs. Rather than drawing from national evidence-based or promising practice registries vetted by a panel of experts, multiple informants reported that program staff identified approaches they felt were relevant through lesser known academic publications, conference presentations, or word of mouth. Several grantees in the 2012 Outreach cohort loosely applied other communities' success stories (often those of former Outreach grantees) to framing, planning, or evaluating their own interventions. 


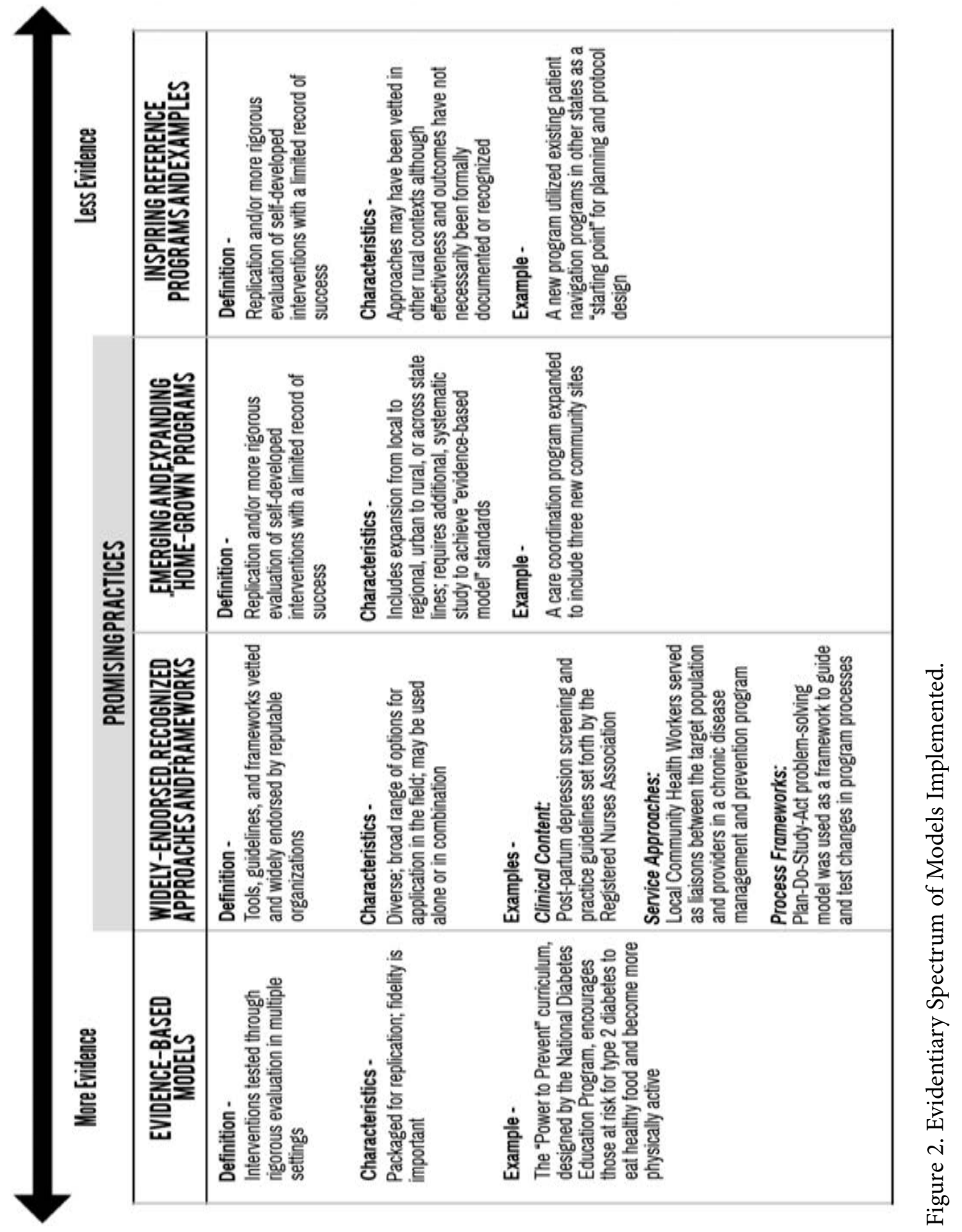


Whether classified as evidence-based programs, promising practices, or inspiring examples, in every case, the grantees reported being purposeful in seeking out and learning from the experiences of other rural and frontier communities. The relative distribution of grantees across the spectrum (Figure 2) is revealing. The vast majority of the cohort employed strategies that fall within the promising practice range, compared to the much smaller subset that applied strictly-defined, evidence-based practices (Figure 2). Grantees attributed this disparity to the limited availability of evidence-based models that had demonstrated effectiveness in rural settings or that seemed feasible in their rural contexts. A similarly small group attempted to translate inspiring, but less well-documented, reference programs from peer communities to their own circumstances (Figure 2). Grantees commonly phrased their view as, "Rural is different," and said that the most relevant programs to examine were those that had been vetted in other rural communities.

Combining models to create hybrid programs. The majority of individual grantees sought to apply multiple models and practices resulting in hybrid or patchwork programs. Grantees varied in what programs they combined, how, and why. Most grantees were very strategic in their combination of concepts and tools. In some instances local leaders had previously developed a promising program, such as care coordination or a mobile clinic, and new evidence-based clinical protocols and practices were added to the existing program. Another approach was to integrate interventions focused on health behavior change with other models for strengthening systems of care and/or facilitating policy, systems, and environmental improvements. Some grantees very carefully chose the most relevant, tested modes of providing services, sharing information, or working with target populations (e.g., telehealth, peer support, home visiting) to introduce research-supported clinical content and meet certain practice guidelines (e.g., nationally recognized standards and guidelines for diabetes, vaccinations, depression, substance abuse, or pediatric oral care).

Barriers to translating evidence-based and promising health practices to rural and frontier settings. Because relatively few evidence-based models have been developed or thoroughly tested in rural contexts, the grantees faced cultural and practical challenges translating otherwise reputable health practices into frontier and rural community settings. When asked to characterize their experience applying models locally, grantees reported varying degrees of difficulty in adaptation and implementation. Common factors complicated implementation across grantee sites.

Cultural misalignment. For many grantees the greatest challenge was a lack of alignment between the programmatic content and the target population. In many cases program materials and clinical recommendations were not appropriate due to low literacy levels, language differences, and high poverty rates seen in many of the grantee communities. Multiple sites reported having to adapt program materials to their targeted audience and local context. Adaptations included shortening and simplifying data collection instruments; modifying language and terminology to a lower reading level; and removing or adjusting program components that were inappropriate given the rural context (e.g., removing healthy food shopping and healthy dining out activities due to transportation challenges and lack of local access in many rural communities). 
Practical limitations. Grantees faced numerous practical obstacles to implementing evidence-based curriculum with community clients and clinical staff. Primary concerns related to the time and financial resources required to conduct and participate in staff training and patient education classes. In remote and frontier areas, travel distances and related costs sometimes precluded participation, especially when multiple sessions were required over an extended period of time.

Lack of practitioner or partner commitment. Because the introduction of new clinical guidelines, standards, and curricula often necessitated changes in mindset or professional practices, grantees frequently met resistance from strategic partners and practitioners, whose support and participation were needed for implementation success. Grantees specifically mentioned the complexity of integrating new approaches into highly structured environments in which treatment, referral, and other protocols are mandated or established by entities outside the implementation site (e.g., hospital systems, federally qualified health centers, public schools).

Insufficient capacity. The successful implementation of many research-based models depends upon finding and training staff for critical and sometimes highly specialized jobs. However, barriers to health care workforce development, recruitment, and retention surfaced as persistent challenges in rural communities.

Unfavorable policy conditions. Some grantees were confronted with significant contextual challenges that were beyond their control. For instance reimbursement policies and requirements related to diabetes testing equipment and remote monitoring undermined patient recruitment for one grantee's telehealth initiative. Similarly, a U.S. Food and Drug Administration policy cautioning against the use of glucometers for fast-turnaround pre-diabetes screening threatened program sustainability by placing additional administrative and equipment use requirements on a different grantee program.

Adapting and tailoring programs to fit the local context. Most grantees reported adapting reference models to overcome challenges and tailor programs to meet community-specific needs and interests. In the Outreach Funding Opportunity Announcement, FORHP allowed grantees significant latitude to adapt selected approaches to overcome challenges and ensure a good fit with local conditions and cultures.

Proposed Outreach projects can take the framework of an evidence-based or promising practice model and tailor it to their community's need and organization. ${ }^{13}$

The relatively small subset of grantees implementing the most rigorously tested, evidence-based models for which fidelity was a paramount concern were less likely than their peers to make ad hoc adjustments in the field. The majority of grantees, however, were less deliberate about fidelity to an original model. Box 1 provides select examples of adaptations made by the 70 case communities to better align programs and practices with their rural and frontier settings. Grantees consistently reported making deliberate, strategic changes in the following areas.

Content. Many Outreach programs involved the use of tested, expert-developed curricula that proved impractical for their rural communities and populations. In such instances, local program directors consolidated the content, added data or informa- 
Box 1.

PROGRAM ADAPTATIONS TO RURAL AND FRONTIER SETTINGS

Types of Adaptation

Adaptation of Program Content

- Condensed educational materials so the information could be shared over an abbreviated time period.

- Compressed content delivered through a workshop series into fewer sessions to reduce travel burden on participants and improve retention.

Adaptation in Modes and Setting

- Adjusted mode of delivery

- Moved program activities to a setting deemed more effective

\section{Adding Wrap-around Components}

- Convened collaborative partnerships specific to subpopulations to help plan and implement programs more effectively.

- Tailored follow-up practices to support clients to reaching their behavior change and health improvement goals.
Examples

- As part of a worksite intervention the Diabetes at Work curriculum shortened from 30-60 minutes to 15-minute lunch and learn sessions because of the short lunch break factory workers were allowed.

- An evidence-based chronic disease management program was shortened from twelve group education sessions to eight.

- Home visits and classroom education series suggested by the promising practice were replaced with monthly phone calls.

- Oral health services provided in a school setting versus a primary care office due to concerns regarding patient transportation and the ability for adequate follow-up.

- One program engaged two different care coordination partners to provide differentiated wrap around services for Hispanic and non-Hispanic clients.

- Examples include regular follow-up calls, in-home visits, or the addition of community health workers to reinforce important messages and maintain adherence to clinical recommendations. 
tion that they noticed were missing, simplified the explanations, and/or changed the frequency and timing of classes in an effort to improve patient retention.

Modes and settings. In addition to modifying the content of curricula, some grantees chose to alter the modes they used to share the information or the settings in which they interacted (e.g., case management follow-up appointments conducted by phone rather than in person due to transportation barriers; preventive screenings and health promotion activities held in the community rather than in clinical settings). These changes were based on community-specific preferences, limitations, and resources.

Wrap-around components. To facilitate uptake among community stakeholders and patient groups, many grantees elected to add connective components or program features to enhance the effectiveness of their promising practices.

Receiving innovation support. Participants reported seeking guidance and assistance from a variety of sources in implementing and adapting their funded programs. Grantees said that mentorship and guidance from evidence-based practice experts or model communities from which programs originated helped them manage and overcome implementation-related obstacles. Multiple grantees described reaching out to peers and program coordinators from other rural communities to learn more about their promising practices and to confer about local adaptations of those practices to rural settings.

As part of the Outreach program, each grantee also received FORHP-funded technical assistance for the three-year grant cycle. Technical assistance providers supported the rural delivery systems in implementing their programs effectively, creating the infrastructure to support funded programs, developing strong partnerships among stakeholders, and planning for the long-term sustainability of the program outcomes and impact. They also facilitated connections with other rural communities implementing similar programs or facing similar challenges (e.g., patient recruitment and retention, provider resistance). Technical assistance related to implementing and adapting promising and evidence-based practices focused on improving efficiency and effectiveness of program approaches (e.g., Community Health Worker models, telehealth), adding or strengthening program elements (e.g., measurement tools used in similar programs, sample partner agreements, program protocols from similar programs), and connecting grantees to resources to assist them in responding to barriers to program implementation.

\section{Discussion}

This study identifies a broad range of dynamics and interacting systems that influence rural practitioners' use of evidence-supported programs and practices to improve health and access to care in their communities. The findings have implications for several constituencies represented in ISF (Figure 1) including funders, researchers, technical assistance providers, and local practitioners. Patterns in the experience of 70 case communities point to the following potential opportunities for building and applying a rural health practice evidence base:

- Funding: Through the Outreach program, FORHP provided funding and incentivized grantees to build on what has been shown to work in other settings to improve 
effectiveness and avoid the need for duplication of efforts. Due to profound local resource limitations, continued external investment is important to supporting the generation and application of evidence-based practices specifically in rural settings.

- Rural-specific research and theory: In most cases, selection and application of evidence-based practices and promising practices were hampered by the fact that few models have been developed and tested specifically in rural contexts. Rigorous study of models originating in and translated to rural contexts will provide a broader evidence base to inform local delivery systems' selection, translation, and adaptation of evidence-based practices. Participatory research partnerships with rural practitioners and patients could be especially instructive.

- Synthesis and translation using a rural lens: Model protocols, materials, and content required significant adaptation to improve their usefulness in the field. Applying a rural lens to examination of existing evidence and packaging it for replication may enhance the relevance of materials and reduce the need for significant adaptions in the field.

- Innovation support: Contextual factors-resource limitations, geographic isolation, poverty, population diversity, literacy rates, persistent workforce shortages, and rural culture-were cited as common obstacles to the implementation of evidence-based practices in rural communities. Technical assistance and training may be needed to support the development of both general and innovation-specific capacities at the delivery system level. General support may include assistance in selecting appropriate models for replication in rural conditions; neutral facilitation to promote partnerships and buy-in; and evaluation training and design. Innovation-specific support could involve consultation regarding fidelity or the strategic adaptation of particular models.

- Rural delivery system: It appears that grantees' intervention selection and adaptation decisions were motivated by a strategic intent to more efficiently and effectively engage target populations and local partners in the process of sustainable health improvement and system change. It is unclear, however, based on the available data whether the adaptations significantly affected program outcomes-positively or negatively - and to what extent they posed a threat to the fidelity of the evidence-based and promising practices they intended to emulate. Prioritizing local program evaluation to document intervention outcomes is necessary to build an evidence base for rural interventions that improve health and strengthen systems of care.

Conclusion. The FORHP's Outreach program created a real-world laboratory for better understanding the dynamics involved in translating evidence-based interventions for use in rural and frontier settings. A broad range of stakeholders are needed to play complementary roles in cultivating a new generation of rural-specific models for improving health and strengthening systems of care. This study points to a need for funders to support programming along a broad spectrum of evidentiary rigor while a more substantial, rural-specific evidence base can be established through systematic research, local evaluation, and robust technical assistance. 


\section{Acknowledgments}

In 2014, The Georgia Health Policy Center was awarded a Direct Services Contract by the Federal Office of Rural Health Policy. The provision of technical assistance services and support to FORHP Community-Based Division grantees are made possible through this multi-year contract.

The research for this manuscript was supported by the Health Resources and Services Administration, Federal Office of Rural Health Policy's, Direct Services Technical Assistance Contract Number HHSH250201000087G.

We thank the former director of the Georgia Health Policy Center's technical assistance team, Beverly Tyler, who provided insight and expertise that greatly assisted this research. We also thank Lori Solomon for offering a thoughtful and methodical review of the manuscript and Attiyya Mujahid Ali for assisting with the design of graphics. Both Lori and Attiyya are staff of the Georgia Health Policy Center.

Portions of this study were presented in poster format at American Public Health Association Conference on November 2, 2015.

\section{References}

1. Wandersman A, Duffy J, Flaspohler P, et al. Bridging the gap between prevention research and practice: the Interactive Systems Framework for dissemination and implementation. Am J Community Psychol. 2008 Jun;41(3-4):171-81.

http://dx.doi.org/10.1007/s10464-008-9174-z

PMid:18302018

2. Flaspohler P, Lesesne CA, Puddy RW, et al. Advances in bridging research and practice: introduction to the second special issue on the interactive system framework for dissemination and implementation. Am J Community Psychol. 2012 Dec;50(3-4):271-81. http://dx.doi.org/10.1007/s10464-012-9545-3

PMid:22875685 PMCid:PMC3766740

3. Centers for Disease Control and Prevention (CDC). Applying science, advancing practice: understanding the Interactive Systems Framework for dissemination and implementation. Atlanta, GA: CDC, 2014. Available at: http://www.cdc.gov/violence prevention/pdf/asap_isf-a.pdf.

4. Castro FG, Barrera M Jr, Martinez CR Jr. The cultural adaptation of prevention interventions: resolving tensions between fidelity and fit. Prev Sci. 2004 Mar;5(1):41-5. http://dx.doi.org/10.1023/B:PREV.0000013980.12412.cd PMid:15058911

5. Meyers DC, Durlak JA, Wandersman A. The quality implementation framework: a synthesis of critical steps in the implementation process. Am J Community Psychol. 2012 Dec;50(3-4):462-80.

http://dx.doi.org/10.1007/s10464-012-9522-x

PMid:22644083

6. Collaborative for Academic, Social, and Emotional Learning (CASEL). Leading an SEL school: steps to implement social and emotional learning for all students. Chicago, IL: CASEL, 2011. Available at: http://www.promoteprevent.org/sites/www .promoteprevent.org/files/resources/leading_an_sel_school.pdf.

7. Hawkins JD, Catalano RF, Arthur MW. Promoting science-based prevention in communities. Addict Behav. 2002 Nov-Dec;27(6):951-76. 
http://dx.doi.org/10.1016/S0306-4603(02)00298-8

8. Saul J, Duffy J, Noonan R, et al. Bridging science and practice in violence prevention: addressing ten key challenges. Am J Community Psychol. 2008 Jun;41(3-4):197-205. http://dx.doi.org/10.1007/s10464-008-9171-2 PMid:18340524

9. Harris JR, Cheadle A, Hannon PA, et al. A framework for disseminating evidence-based health promotion practices. Prev Chronic Dis. 2012;9:E22. Epub 2011 Dec 15. PMid:22172189

10. Chambers DA. The interactive systems framework for dissemination and implementation: enhancing the opportunity for implementation science. Am J Community Psychol. 2012 Dec;50(3-4):282-4.

http://dx.doi.org/10.1007/s10464-012-9528-4 PMid:22688847

11. Noonan RK, Wilson K M, Mercer SL. Navigating the road ahead: public health challenges and the interactive systems framework for dissemination and implementation. Am J Community Psychol. 2012 Dec;50(3-4):572-80. http://dx.doi.org/10.1007/s10464-012-9534-6 PMid:22806091

12. Infante A, Knudson A, Brown A. Promising practices for rural community health worker programs. Chicago, IL: Walsh Center for Rural Health Analysis, 2011. Available at: http://www.norc.org/PDFs/Walsh\%20Center/Rural\%20Evaluation\%20Briefs /Rural\%20Evaluation\%20Brief_March2011_Yseries_1.pdf.

13. Health Resources and Services Administration (HRSA). Rural health care services outreach program; HR-15-039. Washington, DC: HRSA, 2014. Available at: http:// www.hrsa.gov/ruralhealth/programopportunities/fundingopportunities/default.aspx ?id=e325b43e-a8ba-4368-ac25-363f6bab4115. 